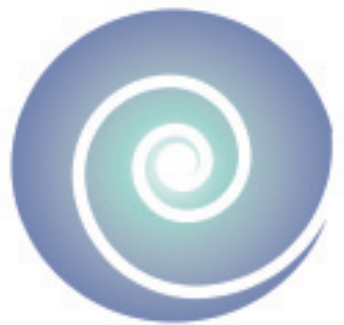

\title{
Treatment of Knee Arthrofibrosis and Quadriceps Insufficiency after Patellar Tendon Repair: A Case Report Including Use of the Graston Technique
}

Douglass W. Black, PT, DPT, SCS, ATC

Duke Physical Therapy and Occupational Therapy, Durham, NC, USA

Arthrofibrosis of the knee is a surgical complication that can limit range of motion, inhibit muscle activity, and decrease patient function. Optimal conservative treatment has not been well established in the literature, leaving a clinician with limited evidence for treatment planning. Described here is part of the rehabilitative course of care for a patient with arthrofibrotic limitations after a mid-substance patellar tendon repair with augmentation. Marked limitations in knee flexion range of motion and quadriceps activity were addressed using the Graston Technique to deal with soft-tissue adhesions; traditional physical therapy care was also provided. Clear improvement in range of motion and quadriceps activity and function was noted over the course of 5 treatments during 1 month. Treatment process and clinical reasoning are offered to promote understanding and to facilitate future inquiry.

KEYWORDS: Rehabilitation of contracture, therapy of joint diseases, manual therapies, musculoskeletal manipulations, tissue adhesions, rehabilitation of postoperative complications, patellar tendon

\section{INTRODUCTION}

"Exaggerated pathologic fibrous hyperplasia" (arthrofibrosis) of the anterior knee soft tissues is described as a debilitating complication of knee surgery ${ }^{(1,2)}$. Its presence can limit physiologic range of motion (ROM), inhibit accessory joint motions, and dampen muscle activation. Most of the arthrofibrosis literature addresses arthrofibrosis after arthroplasty or ligamentous reconstruction, and it is replete with manipulation under anesthesia or with arthroscopic interventions, or both ${ }^{(2-4)}$. There is little treatment-specific evidence to guide more conservative (nonsurgical) management of postoperative knee arthrofibrosis, particularly after patellar tendon repair $^{(5,6)}$. Clinicians are left to gather and apply what are felt to be the most applicable principles.

This report presents a unique case involving a patient who ruptured a patellar tendon. The patient had an unusual type of tear, with a unique surgical approach and two distinct bouts of physical therapy care. Although the patient continued rehabilitation longer than is reported here, the marked progress from initial evaluation through the first month of care is worthy of examination. This case can provide a first reference for clinicians who encounter difficult cases with unique elements and can promote further inquiry.

\section{Patellar Tendon Rupture}

Patellar tendon rupture is a complete disruption of the tendon somewhere between the patella and the tibial tubercle ${ }^{(7)}$. Force transmission from the anterior thigh musculature is lost, thus eliminating the capacity to extend the knee volitionally. Patellar tendon ruptures are uncommon ${ }^{(7,8)}$. One study in a military population delineated 12 patellar tendon failures in 93,224 exposure years. Interestingly, 9 of the 12 injuries occurred while the individual was playing basketball ${ }^{(9)}$.

Loss of active knee extension is devastating to lower extremity (LE) function and needs to be addressed if the ability to ambulate, stand, or perform most LE functions is to be maintained. With repair, most patients return to normal activities of daily living, and many return to the previous level of athletic activity ${ }^{(8,10)}$.

In this case, the initial injury included a midsubstance Z-shaped tear. The surgical resolution incorporated a porcine dermis scaffold to augment the repair. This occurrence was uncommon in both the location of failure and the method used to create a stoutly repaired extensor mechanism.

Without systemic disease involvement, most patellar tendon failures occur at the inferior pole of the patellar. Indeed, a recent case series at a large tertiary medical center included 30 consecutive patellar tendon repairs over an 11-year period and noted that all were "avulsion injuries at the tendon's insertion into the patella"(11). Rupture typically happens with a sudden eccentric contraction of the quadriceps, usually with the foot planted and the knee flexed as the person falls ${ }^{(8,10)}$.

The definitive treatment for this problem is surgical repair with subsequent rehabilitation. The literature is rife with descriptions of surgical technique and 
long-term outcomes. Rehabilitation after surgery typically involves a brief period of immobilization with a gradual return of patellar mobility, knee motion, and strength, followed by systematic return to functional activities, and eventually full return to the activities that are of importance to the patient ${ }^{(12)}$. The most common complications of patellar tendon repair are weakness and loss of motion ${ }^{(12-14)}$.

\section{The Graston Technique}

The Graston technique (GT) is an instrumentassisted soft-tissue mobilization technique. Six stainless steel instruments (TherapyCare Resources, Indianapolis, IN, USA) designed to "adapt to the various tissues/shapes/curves of the body are used to detect and release scar tissue, adhesions and fascial restrictions" (15). The instruments were developed as a transverse friction massage alternative. The stainless steel acts somewhat like a tuning fork when fibrotic tissue is contacted. A resonance or reverberation within the instrument is created on contact and transmits through the instrument to the clinician's hands ${ }^{(15)}$. It is proposed that a clinician's ability to detect adhesions is improved by the vibration transmission of the metal instrument and that adhesion release can be specified by adaptation of instrument surface and treatment stroke ${ }^{(15,16)}$.

The overarching treatment rationale for the GT is based on the manual soft-tissue mobilization rationale proposed by Cyriax ${ }^{(17)}$. Friction massage may be used to promote a local hyperemia, massage analgesia, and reduction of scar tissue ${ }^{(18)}$. In addition, it has been hypothesized that late stages of healing are facilitated to completion by friction ${ }^{(19)}$. Increased fibroblast recruitment has been demonstrated in Graston-type soft-tissue mobilization ${ }^{(20)}$. Application of heavy pressure (compared with light or moderate pressure), using instruments, has also been demonstrated to promote a greater fibroblastic response ${ }^{(19)}$.

One of the concerns that clinicians express with this aggressive soft-tissue work is the potential for excessive tissue damage and ecchymosis. A full discussion of the safety of the GT is beyond the scope of the present report, but needs to be noted as a concern. Hyperplastic scar tissue is by nature excessive and nonfunctional ${ }^{(21)}$. Breakdown of this tissue may allow for functional normalization of the surrounding soft tissues.

The GT is not used in isolation. It is imperative that motion and strengthening be used in conjunction with soft-tissue mobilization to promote tissue adaptation and remodeling. Mechanical load has been shown to affect chondrocyte alignment and fibroblast activity with increased proteoglycan and collagen synthesis ${ }^{(22,23)}$.

\section{Lower Extremity Functional Scale}

The Lower Extremity Functional Scale (LEFS), developed by the North American Orthopaedic
Rehabilitation Research Network, consists of 20 items ${ }^{(24)}$. Each item is scored from 0 (extreme difficulty/unable to perform activity) to 4 (no difficulty), for a total maximum score of 80 points, which indicates a high functional level. The LEFS has been determined to have construct validity and to be concurrent with the SF-36 Health Survey, which served as the principal generic measure for comparison with condition-specific measures ${ }^{(25)}$. For the LEFS, the minimal clinically important difference is 9 scale points [ $90 \%$ confidence interval $(\mathrm{CI})]$, and the minimal detectable change is \pm 9 points $(90 \% \mathrm{CI})$. In a population with LE musculoskeletal dysfunction, the sensitivity of the LEFS to change was superior to that of the SF-36(24).

Every patient being evaluated in our clinic for a LE musculoskeletal diagnosis is asked to complete the LEFS. The scale is used to establish functional baseline status and to inform treatment planning. To facilitate clinical outcomes assessment, LEFS completion is repeated as directed by the physical therapist, but at least once monthly during the patient's course of care. Intervals between LEFS administration are determined by a combination of visit frequency and status change on the part of the patient, and clinical judgment on the part of the practitioner.

\section{Quadriceps Lag}

A quadriceps extension lag (“quad lag") is present when the patient cannot actively extend the knee. Quad lag is determined by the difference between the angle of knee extension achieved passively and the angle at which the patient has volitional control ${ }^{(26)}$. Full active hyperextension, with no quad lag, is a key strength benchmark for progression of strength and functional activities and for gait normalization on level and unlevel surfaces, including stairs.

\section{METHODS}

\section{Case Profile}

A 37-year-old man sustained a non-contact injury to his left knee while playing basketball. He reported that, upon landing from a jump, he felt his left LE slip from underneath him. As he slipped, he felt a sharp pain ( $8 / 10$ using the numeric pain scale) in the anterior left knee area. He was immediately unable to extend his knee, and he reported that he felt that the patella was "out of place" and could be moved easily. His pain resolved quickly (within 5 minutes) to $0 / 10$, but he was unable to weight bear or ambulate on the left LE.

The patient was transported immediately after the injury to a local level 1 trauma center. After a triage screening, including an extensive medical history, plain radiographs, and a physical exam, it was 
determined that the patellar tendon was ruptured. Arrangements were made for surgical repair within 48 hours of the initial insult. The patient had no history of previous tendinopathy, anabolic steroid use, renal failure, or systemic disease.

At the time of surgery, the injury was found to be in the mid-substance rather than at the inferior patellar pole (the more typical failure). It was reported that there was "no obvious tendinopathy such as calcification or chronic changes" to suggest any prior patellar tendonitis. All other ligamentous and cartilaginous structures were found to be intact and pristine. Because of the mid-substance, Z-shaped nature of the patellar tendon tear, the orthopedic surgeon added a porcine dermis graft (Conexa 200: Tornier, Edina, MN, USA) for repair augmentation (see Figs. 1 and 2).

The patient was permitted to perform patellar mobilization and quad sets on the day of surgery, but

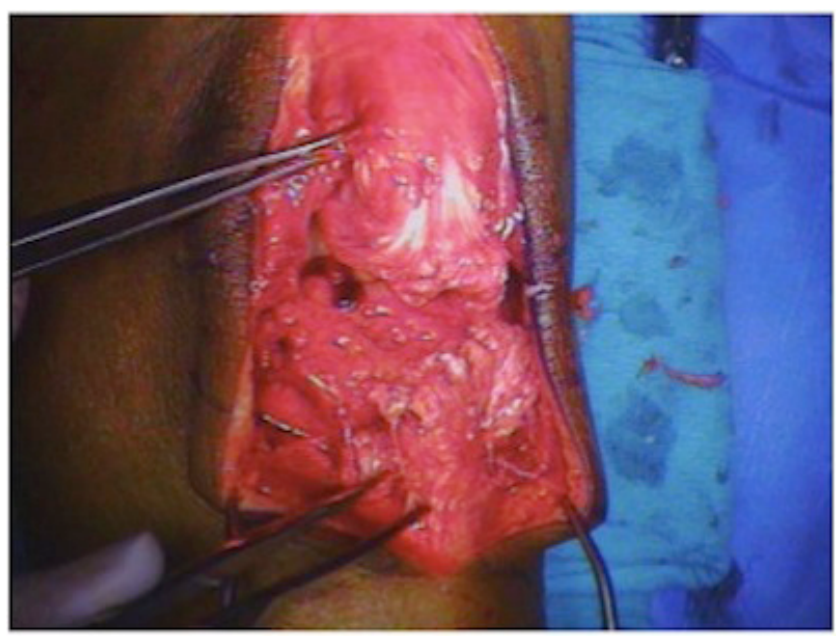

FIGURE 1. Perioperative photo of Z-shaped mid-substance patellar tendon tear before repair and augmentation.



FIGURE 2. Completed patellar tendon repair with porcine scaffold augmentation. was not allowed any ROM activities for 7 days postoperatively. Once allowed, initial ROM restrictions were $0-60$ degrees as tolerated. He began physical therapy 4 days postoperatively near his home, about $160 \mathrm{~km}$ distant from our facility, and he was treated at that clinic for just over 9 weeks. Formal rehabilitation sessions occurred $2-3$ times weekly in addition to the daily home exercises. The patient also used a continuous passive motion device for $2-6$ hours daily, at home, beginning at 7 days after surgery and continuing until his 10-week follow-up visit, when its use was discharged.

Although detailed specifics of this man's initial rehabilitative course were not available to our clinic, modality and activity types were conveyed. Initial physical therapy included modalities for pain and edema control, patellar mobilization, quad sets, ankle ROM, and strengthening activities, until knee ROM was also included per precautions. Electrical stimulation was used to promote quadriceps activity, edema reduction, and pain control. Patellar mobilization were performed by the clinician and assigned as part of the patient's home exercise program. Once permitted, ROM activities included passive range of motion (PROM) performed manually by the clinician and active assisted range of motion (AAROM) performed by the patient with assistance from the contralateral limb or an external device such as a towel or belt. The AAROM activities were included in the home exercise program, which the patient was instructed to perform 3- 5 times daily. Quad sets, ankle ROM, and strengthening activities such as active knee extension, terminal knee extension against band resistance, and resisted hamstring activities were used in the clinic and as part of the home exercise program. The patient reported that emphasis was placed on performing quad sets up to 10 times daily.

At the 2-week postoperative visit, 40 degrees of passive knee flexion, with extension to 0 degrees, was demonstrated. Straight-leg raise activities in the locked postoperative brace were permitted at this point, and the knee flexion ROM restriction was modified to 90 degrees. At the subsequent 6-week visit, 85 degrees of flexion was demonstrated, and the ROM limit was moved to 120 degrees of flexion. It was expected that 120 degrees of flexion would be achieved no later than the next surgical follow-up at the 10-week mark.

Unfortunately, the patient demonstrated a 25- to 30-degree quad lag and approximately 90 degrees of flexion ROM at the 10-week follow-up. These findings were decidedly below the expected level of recovery and thus prompted referral to our clinic to help ameliorate the limitations. At this juncture, ROM restrictions were lifted, and the patient was instructed that he needed to ambulate with at least one crutch and the postoperative knee brace on, and he was restricted to 60 degrees of flexion during gait until he demonstrated a quad lag of less than 5 degrees. 


\section{Physical Therapy Evaluation}

\section{Subjective Assessment}

After a review of all accessible information regarding the patient's injury, surgical intervention, and rehabilitative course to date, a physical therapy evaluation was performed at our facility. In discussion, the patient confirmed the history previously noted, denied any other significant past medical or surgical history, and reported an excellent general health status. Using a numeric pain scale, he reported pain to be $0 / 10$ at the time and at best, but $5 / 10$ at worst. The patient arrived to his appointment ambulating with partial weight-bearing on the left LE, with bilateral axillary crutches and the postoperative brace limited to 60 degrees of flexion.

The patient reported that, when he did have pain, it was sharp in nature and located in the left knee around the surgical incision sites and at the distal portion of the quadriceps. The only aggravating factor reported was movement of the involved joint. No alleviating factors were delineated. The patient also noted that, before the injury, his level of function was independent and without limitations. His LEFS score was $18 / 80(23 \%)$, with a global function rating of $50 \%$ for activities of daily living and $0 \%$ for sports and recreation activities.

Work demands were reported to include standing for extended periods and extensive travel by car and airplane. The patient noted that, before the injury, he ran $1-3$ miles for exercise almost daily and played basketball up to 4 times weekly. These work and recreation activities formed the basis of his selfreported short- and long-term goals. Self-reported goals included the ability to tolerate standing without assistance for up to 4 hours at a time and an eventual return to running and basketball activities.

\section{Objective Examination}

The patient was noted to have full ROM of the right knee and full and equal ROM of the bilateral hips and ankles. For the left knee, PROM was measured at 95 degrees of flexion and 2 degrees of hyperextension. Active ROM (AROM) for the left knee was 93 degrees of flexion, with 20 degrees of extension. The difference between active and passive left knee extension constituted a quad lag of 22 degrees. All other strength measurements were deferred.

During gait evaluation, the patient ambulated with bilateral axillary crutches using a 2-point gait in which the crutches moved in concert with the affected limb. He was weight-bearing as tolerated during this activity and demonstrated a right lateral lean, left hip circumduction or hiking, decreased gait velocity, and left LE external rotation at stance. No assistance was required for safe level-surface ambulation.

The surgical wound on the left LE was well healed, and moderate generalized left knee swelling was present. On visual inspection, marked scar proliferation was noted around the incision site. On palpation, tenderness was detected along the patellar tendon incision site of the left knee, the quadriceps tendon, the distal third of the quadriceps (including the musculotendinous junction), and along the medial and lateral patellar borders. Adhesions of a thick, immobile nature were noted throughout the anterior knee region, being most prominent at the surgical incision sites, the suprapatellar pouch, and the infrapatellar fat pad soft tissues. The skin was adheased to the deeper layers along the incisional scar and within approximately $1 \mathrm{~cm}$ of the scar. No crepitus was noted with manual patellar movement, and the total physiologic and accessory movement of the left patella was approximately $25 \%$ of that found on the right.

The borders of the rectus femoris on the vastii were limited to medial and lateral movement with manual distention and demonstrated little to no proximal movement with quadriceps activation. Adhesions were palpable along the medial and lateral borders of the rectus femoris from the suprapatellar pouch area until approximately $5 \mathrm{~cm}$ distal to the muscular insertion at the anterior inferior iliac spine (AIIS). In addition, as the patient was asked to plantar-flex the left ankle, he reported a pulling sensation, or tightness, at and for approximately $3 \mathrm{~cm}$ distal to the tibial tubercle. These proximal and distal findings suggested that soft-tissue restrictions were not limited to local knee adhesions, but followed the myofascial superficial front line (SFL) as described by Myers ${ }^{(27)}$. The SFL includes the tibialis anterior, the extensor mechanism, and the quadriceps, particularly the rectus femoris, all of which demonstrated adhesion in this case.

Based on the foregoing examination and discussions with the patient, a decision was made to focus on soft-tissue adhesion release, flexion ROM, and quadriceps strength and control. Gait was determined to be a secondary consideration, to be addressed only as dictated by improvement in the primary parameters.

\section{Goals}

Short-term goals set at the initial evaluation included full active hyperextension within 6 weeks and left knee ROM symmetrical to the right within 8 weeks. Long-term goals included left LE strength equal to the uninvolved side in 4 months, LEFS score of $90 \%$ or greater in 5 months, and full return to running and basketball in 8 months.

\section{Treatment Plan: Interventions}

The patient was seen for 5 visits over a 4-week period with treatment in each session following a similar pattern: warm-up, soft-tissue mobilization, patellar or tibiofemoral joint mobilization (or both), flexion ROM activities, strengthening activities, modalities for edema control, and home exercise program assignment, review, and progression. 


\section{Warm-Up and Soft-Tissue Mobilization}

Warm-up was done with a moist hot pack applied over the knee for 5-7 minutes. Soft-tissue mobilization using the GT was performed immediately after the heat was removed. The GT was applied to both the prominent adhesions locally and along the length of the SFL from the ankle retinaculum to the AIIS (see Figs. 3 and 4). Initial or scanning strokes were superficial in nature, with intent to feel for all areas of adhesion. The location and quality of the adhesions in terms of size, rigidity or pliability, and tenderness to instrument application were noted. Brief bouts $(30-60 \mathrm{~s})$ of deeper and more specific instrument application in all directions over each area of interest followed. Specific locations of the deeper application were different at each visit. Focal areas of marked adhesion included the suprapatellar pouch, the medial and lateral patellar borders, the infrapatellar fat pad, and the rectus femoris and proximal tibialis anterior muscles.

For the first 2 visits, the GT was applied with the patient supine or long-sitting with a small towel roll

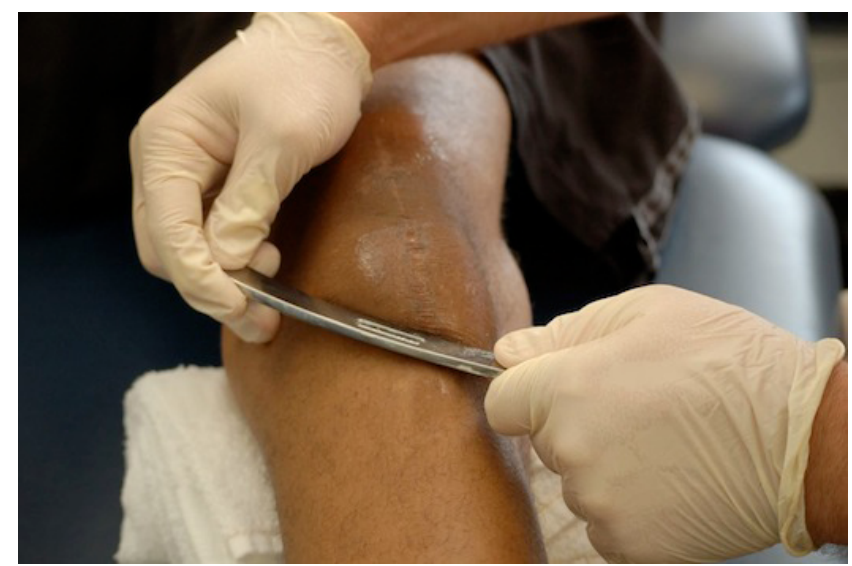

FIGURE 3. Graston technique: instrument application to the patellar tendon and surgical incision site.

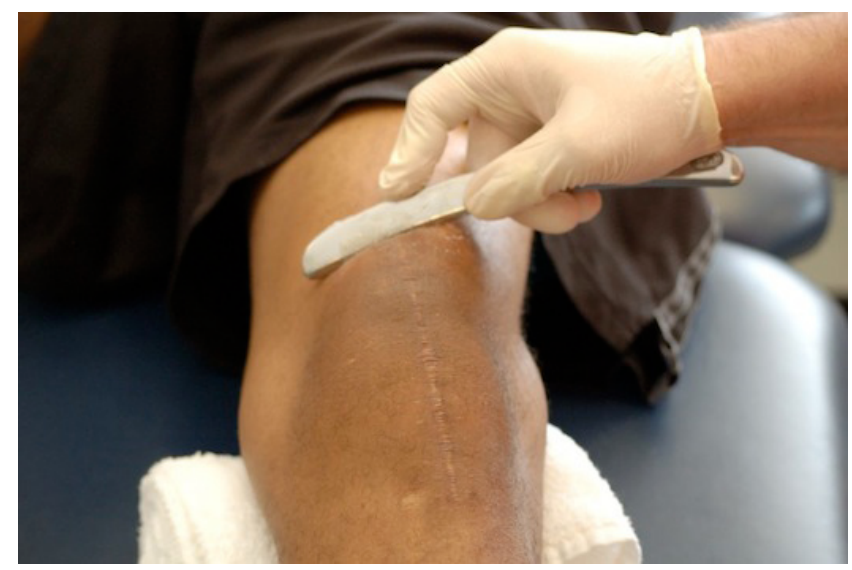

FIGURE 4. Graston technique: instrument application to the distal quadriceps and suprapatellar pouch. The patient actively extends and flexes the knee during treatment to augment soft-tissue release. (5-cm diameter) under the left knee. For the next 3 visits, GT scanning was performed with the patient passive on the plinth, while adhesion-specific treatment was performed with the patient moving his knee, activating underlying musculature, or performing functional activities-for example, closed-chain terminal knee extensions (TKEs), mini-squats. During the scanning process at visit 3 , it was felt that the more superficial adhesions were resolving well and that the deeper structures would be better addressed with activation or function. Activities performed after the GT were chosen with intent to support fascial and scar release and to improve volitional control of the quadriceps and other SFL musculature of the LE.

\section{Joint Mobilization and ROM}

Patellar joint mobilizations of Maitland grades III and IV were performed in the medial, lateral, superior, and inferior directions using 30 oscillations each time ${ }^{(26)}$. Tibiofemoral mobilizations were of similar quality to those for the patella, but were performed in the anterior and posterior directions. The ROM activities included

- PROM in a supine or seated position, with the author performing the motion and a 5-s hold for each repetition;

- use of a stationary bike for ROM, with the patient rocking through the available left-knee ROM by motion of the right LE and holding a 5-s stretch at end range in both the forward and backward directions; and

- $\quad$ seated or supine patient-centered AAROM knee flexion with a belt or contralateral limb assisting the motion into a stretch with a 5-s hold.

\section{Strengthening}

Strengthening activities included only quad sets and concentric and eccentric short-arc quadriceps (SAQ) for the first 2 visits.

During visits $1-4$, the eccentric SAQ were performed concomitantly with application of Russian stimulation in a 10-s on, 30-s off cycle, with a 2 -s ramp time and a $50 \%$ duty cycle. Two pads $(5 \times 13 \mathrm{~cm})$ were placed diagonally from superolateral to inferomedial approximately $5-7 \mathrm{~cm}$ distal to the AIIS and just proximal to the quadriceps tendon such that the distal portion of the lower pad ended over the distal vastus medialis oblique muscle fibers. Intensity was set at the minimum level to produce a tetanic contraction. The patient was encouraged to actively contract the quadriceps during the on portion of each cycle and to rest the muscle for the off portion of the cycle. Use of Russian stimulation was chosen to enhance quadriceps recovery and thus speed resolution of the patient's quad $\operatorname{lag}^{(28,29)}$.

During visit 4, mini-squats were added to the strengthening activities, with emphasis on the eccentric portion of the activity. 
At visit 5, the patient tolerated the addition of TKEs against moderate-to-heavy band resistance and forward/retro walking against $13.5 \mathrm{~kg}$ resistance from a cable column. With each activity, emphasis was placed on quality of motion and control of muscular activity.

\section{Edema and Pain Control}

Near the conclusion of visit 1 , the patient was placed in a supine position on a plinth with elevation of the legs to approximately 45 degrees from horizontal. Four electrical pads $(5 \times 10 \mathrm{~cm})$ were applied around the knee such that the superomedial and inferolateral pair of pads were parallel to each other; the superolateral and inferomedial pads were similarly placed. An interferential current using 2 channels was attached to the pads ( 1 channel for each pair of pads). The interference pattern was set at a frequency range of $1-55 \mathrm{~Hz}$ so that the muscle contractions produced would be sub-tetanic. An ice pack was strapped over top of the knee, and the current was initially set at a strong, but not painful, intensity as determined by the patient. Treatment time was set for 15 minutes. The patient had full control of the intensity level for the duration of treatment.

At all subsequent visits, interferential current was not used, because the edema found at the initial visit was not present. However, at the conclusion of each visit, an ice pack was flexi-wrapped to the patient's left knee, and the patient was instructed to remove it after 20 minutes. This treatment met the need to limit post-activity inflammatory processes while being sensitive to the patient's need to return to work or home in a timely manner.

\section{Home Exercise Program}

All home exercise activities were similarly designed to increase ROM and muscular control. At the initial visit, the patient agreed to a home exercise program consisting of patellar mobilizations, knee flexion PROM, quad sets, and gait practice with use of a mirror for visual feedback. Mobilization and ROM activities were to be performed $3-5$ times daily, with gait practice at least once daily and a session of quad sets performed once each waking hour. This program was reviewed for correct technique and patient follow-through at each visit.

Gait activities were modified to use of a single axillary crutch, and the brace was unlocked to 90 degrees for ambulation. At visit 4, mini-squats were added (to be performed $1-2$ times daily), and gait activities were again modified, this time to no assistive device. Single and double leg presses, hamstring curls, calf raises, TKEs, stationary cycle for cardiovascular activity, and treadmill retrowalking were added at visit 5 . In addition, the patient was instructed to remove the postoperative brace for all activities except when in crowds or faced with the need to stand for more than 1 hour at a time.

\section{Treatment Plan: Measurement}

Because the major deficits exhibited by the patient included not only knee flexion stiffness and quadriceps weakness, but also a marked functional deficit, relevant clinical tools were selected to assess clinical progress. Measurements were taken at visits 1, 3, and 5. Pain was also measured (this being the standard procedure for each patient at each visit to the clinic). Objective measures included standard goniometric knee flexion ROM, both passive and active, and quadriceps strength and control as determined by quad $\operatorname{lag}^{(26)}$. A numeric pain scale from 0 (no pain) to 10 (intolerable pain) was used at each visit. The LEFS was administered on the same visits during which the objective measures were taken.

\section{RESULTS}

At each visit, the patient verbally described improvement in ROM, strength, and function as noted by improved activities of daily living. He also reported performance of the home exercise activities at $80 \%$ of the requested frequency. Pain was noted at the initial visit (as indicated earlier) and at the fourth visit. At visit 4 , the patient reported a pain level of $2 / 10$ in the left knee and noted that it was achy in nature. At the end of that session, he reported that the pain had fully resolved. No pain was reported at any other visit. Functionally, the patient's LEFS score improved from $23 \%$ at evaluation to $32 \%$ at visit 3 and to $44 \%$ at visit 5 . In short, the patient reported that his functional ability had nearly doubled over the reported month of care.

Objectively, the patient's quad lag improved from 22 degrees at evaluation to 8 degrees at visit 3 and then to 3 degrees at visit 5 . His knee flexion AROM was 93 degrees, 103 degrees, and 110 degrees at visits 1,3 , and 5 respectively; and his PROM was noted to be 95 degrees, 108 degrees, and 123 degrees at those same visits. Table 1 summarizes the recorded ROM, strength, and function data.

Gait was not formally measured at each visit, but by visit 5 , the patient was able to ambulate without a brace or assistive device, and he demonstrated a

TABLE 1. Clinical Outcome Measures after Treatments 1, 3, and 5

\begin{tabular}{lccc}
\hline & \multicolumn{3}{c}{ Treatment } \\
& 1 & 3 & 5 \\
\hline Quadriceps lag (degrees) & 22 & 8 & 3 \\
Flexion AROM (degrees) & 93 & 103 & 110 \\
Flexion PROM (degrees) & 95 & 108 & 123 \\
LEFS score (\%) & 23 & 32 & 44 \\
\hline
\end{tabular}

$\mathrm{AROM}=$ assisted range of motion; $\mathrm{PROM}=$ passive range of motion; LEFS = Lower Extremity Function Scale. 
normalized level-surface gait pattern. Similarly, left patellar physiologic and accessory motion was not formally measured at each visit, but at visit 5 was found to be approximately $75 \%$ of the right.

\section{DISCUSSION}

Postsurgical knee-flexion arthrofibrosis is a difficult problem, particularly when the initial injury and subsequent surgical intervention are uncommon in their own right. Basic physical therapy techniques may be insufficient to facilitate adequate patient progression.

In this case, all treatments used were common to traditional physical therapy, with the exception of the choice to use the GT as the specific mode of soft-tissue mobilization. Addition of the GT may have made a difference in clinical and functional improvement measures and allowed for decreased visit frequency. The patient was better able to obtain physiologic and accessory motions of the patellofemoral and tibiofemoral joint after release of soft-tissue adhesions. In turn, this achievement may have reduced inhibition of the quadriceps musculature and allowed for more normal patellar and soft-tissue motion during muscular activity.

During the reported course of care, the patient was seen $50 \%$ to $67 \%$ less frequently, and yet he doubled his reported functional status. He gained 28 degrees of PROM and 17 degrees of AROM, while decreasing quad lag by 19 degrees. By the last visit reported, the patient was able to ambulate on level surfaces without assistive devices or a brace. It is recognized that only short-term results are reported here, but the differences accomplished are notable given the relatively small number of visits compared with his earlier rehabilitative course. The need to gather long-term functional and clinical data is recognized.

The success of this case in dealing with postsurgical adhesions and muscle inhibition, using physical therapy incorporating the GT, provides a first reference for other clinicians who encounter similar issues. Future case and series reports will help to enrich the literature and to provide a resource for clinicians dealing with this difficult problem.

\section{CONFLICT OF INTEREST NOTIFICATION}

The author declares that an honorarium has been received from TherapyCare Resources, Inc., for activities unrelated to this case report.

\section{COPYRIGHT}

Published under the CreativeCommons AttributionNonCommercial-NoDerivs 3.0 License.

\section{REFERENCES}

1. Millett PJ, Wickiewicz TL, Warren RF. Motion loss after ligament injuries to the knee. Part I: causes. Am J Sports Med. 2001; 29(5):664-675.

2. Paulos LE, Rosenberg TD, Drawbert J, Manning J, Abbott P. Infrapatellar contracture syndrome. An unrecognized cause of knee stiffness with patella entrapment and patella infera. Am J Sports Med. 1987; 15(4):331-341.

3. Bong MR, Di Cesare PE. Stiffness after total knee arthroplasty. J Am Acad Orthop Surg. 2004; 12(3):164'-171.

4. Parisien JS. The role of arthroscopy in the treatment of postoperative fibroarthrosis of the knee joint. Clin Orthop Rel Res. 1988; (229):185-192.

5. Millett PJ, Wickiewicz TL, Warren RF. Motion loss after ligament injuries to the knee. Part II: prevention and treatment. $\mathrm{Am}$ J Sports Med. 2001; 29(6):822-828.

6. Noyes FR, Mangine RE, Barber SD. The early treatment of motion complications after reconstruction of the anterior cruciate ligament. Clin Orthop Rel Res. 1992; (277):217-228.

7. Matava MJ. Patellar tendon ruptures. J Am Acad Orthop Surg. 1996; 4(6):287-296.

8. Ramseier LE, Werner CM, Heinzelmann M. Quadriceps and patellar tendon rupture. Injury. 2006; 37(6):516-519.

9. White DW, Wenke JC, Mosely DS, Mountcastle SB, Basamania CJ. Incidence of major tendon ruptures and anterior cruciate ligament tears in US Army soldiers. Am J Sports Med. 2007; 35(8):1308-1314.

10. Scuderi GS, Easley ME. Quadriceps and patellar tendon disruptions. In: Scott WN, ed. Surgery of the Knee. 3rd ed. Philadelphia, PA: WB Saunders; 2001: 1074-1086.

11. West JL, Keene JS, Kaplan LD. Early motion after quadriceps and patellar tendon repairs: outcomes with single-suture augmentation. Am J Sports Med. 2008; 36(2):316-323.

12. Marder RA, Timmerman LA. Primary repair of patellar tendon rupture without augmentation. Am J Sports Med. 1999; 27(3):304-307.

13. Lindy PB, Boynton MD, Fadale PD. Repair of patellar tendon disruptions without hardware. J Orthop Trauma. 1995; 9(3):238-243.

14. Scott WN, Insall JN. Extensor mechanism injuries. In: Rockwood CA Jr, Green DP, eds. Fractures in Adults. Philadelphia, PA: JB Lippincott; 1991: 1816-1839.

15. Graston Technique Module-1 Manual. Indianapolis, IN: TherapyCare Resources; 2006.

16. Hammer WI, Pfefer MT. Treatment of a case of subacute lumbar compartment syndrome using the Graston technique. J Manipulative Physiol Ther. 2005; 28(3):199-204.

17. Stasinopoulos D, Johnson MI. Cyriax physiotherapy for tennis elbow/lateral epicondylitis. Br J Sports Med. 2004; 38(6):675-677.

18. Norris CM. Sports Injuries: Diagnosis and Management. New York, NY: Butterworth-Heinemann: 1993.

19. Gehlsen GM, Ganion LR, Helfst R. Fibroblast responses to variation in soft tissue mobilization pressure. Med Sci Sports Exerc. 1999; 31(4):531-535.

20. Davidson CJ, Ganion LR, Gehlsen GM, Verhoestra B, Roepke JE, Sevier TL. Rat tendon morphologic and functional changes resulting from soft tissue mobilization. Med Sci Sports Exerc. 1997; 29(3):313-319. 
21. Grefte S, Kuijpers-Jagtman AM, Torensma R, Von den Hoff JW. Skeletal muscle development and regeneration. Stem Cells Dev. 2007; 16(5):857-868.

22. Eastwood M, McGrouther DA, Brown RA. Fibroblast responses to mechanical forces. Proc Inst Mech Eng H. 1998; 212(2):85-92.

23. Toyoda T, Saito S, Inokuchi S, Yabe Y. The effects of tensile load on the metabolism of cultured chondrocytes. Clin Orthop Relat Res. 1999; (359):221-228.

24. Binkley JM, Stratford PW, Lott SA, Riddle DL. The Lower Extremity Functional Scale (LEFS): scale development, measurement properties, and clinical application. North American Orthopaedic Rehabilitation Research Network. Phys Ther. 1999; 79(4):371-383.

25. Martin DP, Engelberg R, Agel J, Swiontkowski MF. Comparison of the Musculoskeletal Function Assessment questionnaire with the Short Form-36, the Western Ontario and McMaster Universities Osteoarthritis Index, and the Sickness Impact Profile health-status measures. J Bone Joint Surg Am. 1997; 79(9):1323-1335.
26. Magee DJ. Orthopedic Physical Assessment. 4th enhanced ed. St. Louis, MO: Saunders Elsevier; 2006.

27. Myers TW. Anatomy Trains: Myofascial Meridians for Manual and Movement Therapists. 2nd ed. Edinburgh: Churchill Livingstone; 2009.

28. Snyder-Mackler L, Delitto A, Stralka SW, Bailey SL. Use of electrical stimulation to enhance recovery of quadriceps femoris muscle force production in patients following anterior cruciate ligament reconstruction. Phys Ther. 1994; 74(10):901-907.

29. Mintken PE, Carpenter KJ, Eckhoff D, Kohrt WM, Stevens JE. Early neuromuscular electrical stimulation to optimize quadriceps muscle function following total knee arthroplasty: a case report. J Orthop Sports Phys Ther. 2007; 37(7):364-371.

Corresponding author: Douglass W. Black, Duke Department of Physical Therapy and Occupational Therapy, DUMC Box 3965, Durham, NC, 27710, USA.

E-mail: dpt.scs.atc@gmail.com 